\title{
Mixed Dissociative and Molecular Adsorption of Water on the Rutile (110) Surface
}

\author{
Philip J. D. Lindan and N. M. Harrison \\ Daresbury Laboratory, Warrington WA4 4AD, United Kingdom \\ M. J. Gillan \\ Physics Department, Keele University, Staffordshire ST5 5BG, United Kingdom
}

(Received 12 August 1997)

\begin{abstract}
Using first-principles density-functional methods we show that a monolayer of water on the rutile (110) surface contains $\mathrm{H}_{2} \mathrm{O}$ in both molecular and dissociated forms. Intermolecular hydrogen bonding stabilizes this configuration with respect to the complete dissociative adsorption which would be predicted from studies at lower coverage. The proposed mixed adsorption mode is fully consistent with experimental data, reconciles apparent conflicts within these data, and explains discrepancies between experiment and previous calculations. [S0031-9007(97)05099-0]

PACS numbers: 68.45.Da, 82.30.Nr, 82.65.Pa
\end{abstract}

The adsorption of water on oxide surfaces is poorly understood [1], despite a great deal of investigation. The question is highly relevant: The performance of gas sensors, the weathering of rocks, and the rheology of powders are some of the diverse situations in which water chemistry at oxide surfaces is of central importance. Titanium dioxide, widely used in powder form as a pigment and opacifier, has attracted particular attention. Initially this derived from the observation of the photoelectrolysis of water on rutile $\mathrm{TiO}_{2}$ surfaces [2], but it rapidly became apparent that a wealth of scientific challenge is present in the study of $\mathrm{TiO}_{2}$ surface chemistry. From experiment, it has variously been proposed that, on the (110) surface, water adsorbs molecularly and only dissociates at defect sites [3], dissociatively at low coverages and thereafter molecularly [4], either molecularly or dissociatively depending on temperature [5], or that the surface is inert [1]. One point of consensus is that, if dissociation does occur, it is only at low coverages. In contrast, theorists are unanimous in predicting dissociation at all coverages [6-11]. Such inconsistencies dog the current understanding of oxide surface chemistry.

In this Letter we describe first-principles calculations of water adsorption on the rutile (110) surface. These are the first calculations on this system in which the consequences of interactions between water molecules have been explored. It is shown that this is essential because of strong intermolecular hydrogen bonding, and leads to the prediction of a novel adsorbate geometry in which a monolayer (ML) of water contains $\mathrm{H}_{2} \mathrm{O}$ in both dissociated and molecular forms. This model allows us to reinterpret experimental results and resolve the apparent contradictions they contain, as well as explaining why previous calculations fail to predict the presence of molecular $\mathrm{H}_{2} \mathrm{O}$ at any coverage. The plane-wave pseudopotential densityfunctional theory (DFT) techniques we use [12] allow the prediction of chemical processes from first principles, i.e., simply from the quantum-mechanical behavior of valence electrons. They yield accurate total energies [13], allow- ing the comparison of adsorption mechanisms, and since ionic forces are easily computed, both large-scale structural relaxation and molecular dynamics (MD) simulations are possible [14]. All of these attributes are essential for the present purpose. The utility of this approach for $\mathrm{TiO}_{2}$ has been illustrated in previous studies of bulk [15] and surface $[16,17]$ properties, and surface reconstructions $[18,19]$.

The infrared spectra of water on microcrystalline rutile powders [20] have been interpreted as evidence for two types of $\mathrm{OH}$, and hence for dissociation, on the (110) surface. Ultraviolet photoemission spectroscopy (UPS) of single crystals by Kurtz et al. [5] showed dissociative adsorption on the perfect surface at $300 \mathrm{~K}$, which saturated at $\sim 0.1 \mathrm{ML}$. At $160 \mathrm{~K}$ molecular adsorption was found, which saturated at one ML; subsequent heating produced dissociation of some of the adsorbed molecules. While $\mathrm{O}$ defects enhanced the rate of dissociative adsorption, they did not affect the saturation coverage. Hugenschmidt et al. [4] used x-ray photoemission spectroscopy and work-function measurements to assign a temperatureprogrammed desorption (TPD) peak at $275 \mathrm{~K}$ to molecular adsorption at fivefold-coordinated $\mathrm{Ti}$ sites. Using the UPS results of Kurtz et al. these authors attributed a hightemperature tail extending to $375 \mathrm{~K}$ to $\sim 0.25 \mathrm{ML}$ of dissociatively adsorbed water. Henderson [3] found TPD features much like those of Hugenschmidt et al. and interpreted them similarly. However, he argues that, at $130 \mathrm{~K}$, water dissociation occurs only at $\sim 0.1 \mathrm{ML}$ coverage, and suggests that this is at defect sites. He also used features in high-resolution electron-energy-loss spectroscopy (HREELS) spectra, in particular, a feature at $1625 \mathrm{~cm}^{-1}$ identified as the water $\mathrm{H}-\mathrm{O}-\mathrm{H}$ bond-bending mode, and features around $3400 \mathrm{~cm}^{-1}$ assigned to molecular $\mathrm{O}-\mathrm{H}$ stretch modes, to support the view that the majority of firstlayer adsorption was molecular, especially because the two distinct $\mathrm{OH}$ frequencies expected from dissociated $\mathrm{H}_{2} \mathrm{O}$ were not observed.

Semiempirical tight-binding studies of single-molecule adsorption and hydroxylation [6-8] have been performed, 
and these address both ML and low coverage. Dissociation is favorable in all cases, but these methods cannot reliably predict the energetics involved. Also, only one of these studies included any structural relaxation [8], and all were confined to highly symmetric adsorbate configurations. First-principles calculations at ML coverage have also been reported: from both Hartree-Fock [9] and DFT [10] studies it was concluded that water adsorbs dissociatively on the (110) surface. It is important to note that in all of these theoretical studies, the neighbors of the $\mathrm{H}_{2} \mathrm{O}$ in the simulation cell are its periodic images, and are therefore constrained to be at fixed separation (the cell dimensions in [001] and [1 $\overline{10} 0]$ ) and to have the same orientation. This restriction is a serious one, since on this surface the separation of $\sim 3 \AA$ along [001] may lead to strong intermolecular hydrogen bonding [21]. Finally, we have reported first-principles calculations at half-ML coverage [11]. We found that water adsorbs dissociatively at this coverage, and also demonstrated that the $\mathrm{H}$ vibrational spectrum contains only one sharp, high-frequency peak, contributed by one of the $\mathrm{OH}$ groups. The other $\mathrm{OH}$ contributes in a broad range of frequencies because of hydrogen bonding between the adsorbed $\mathrm{OH}$ and $\mathrm{H}$. This explained some features of the observed spectra, but not the presence of a clear bond-bending signal in the HREELS data and other strong evidence for molecular adsorption at ML coverage.

The technical details of the present Letter are as follows. We used the gradient-corrected Perdew-Wang exchange-correlation functional (PW II [22]). Gradient corrections $[23,24]$ are needed for the accurate treatment of hydrogen bonds, and their inclusion leads to successful descriptions of, e.g., the structure of water [25] and ice [26] and the dissociation energies of small molecules [27]. A $750 \mathrm{eV}$ cutoff was used with optimized, normconserving $\mathrm{Ti}$ and $\mathrm{O}$ pseudopotentials. The construction and testing of these pseudopotentials have been described elsewhere [19]. A slab of ions in a 3D supercell was used to model the surface. The supercell is double the dimension of the surface unit cell in the [001] direction, as in Fig. 1. In the supercell, the (110) slab faces are separated by a vacuum gap of $6.63 \AA$. Two slab thicknesses were considered: 3 layers (36 ions) for MD and 5 layers (60 ions) for static calculations. Brillouinzone sampling was at the $\Gamma$ point for $\mathrm{MD}$, and at two Monkhorst-Pack points for the statics, with no sampling along the surface normal. The increases in slab thickness and $k$-point sampling were made to allow very accurate computation of adsorption energy differences [28]. For the dynamics, the $\mathrm{H}$ mass was set to $3 \mathrm{amu}$, allowing us to use a time step of $1.5 \mathrm{fs}$. This does not affect configuration energies or equilibrium statistical averages such as vibrational mean-square displacements, but does mean that the frequencies we measure must be scaled by a factor of 1.61-1.67 for comparison with experiment [11]. A mass of 3 amu lowers the $\mathrm{H}_{2} \mathrm{O}$ angle-bending frequency to $29 \mathrm{THz}$, still well clear of the $\mathrm{TiO}_{2}$ lattice

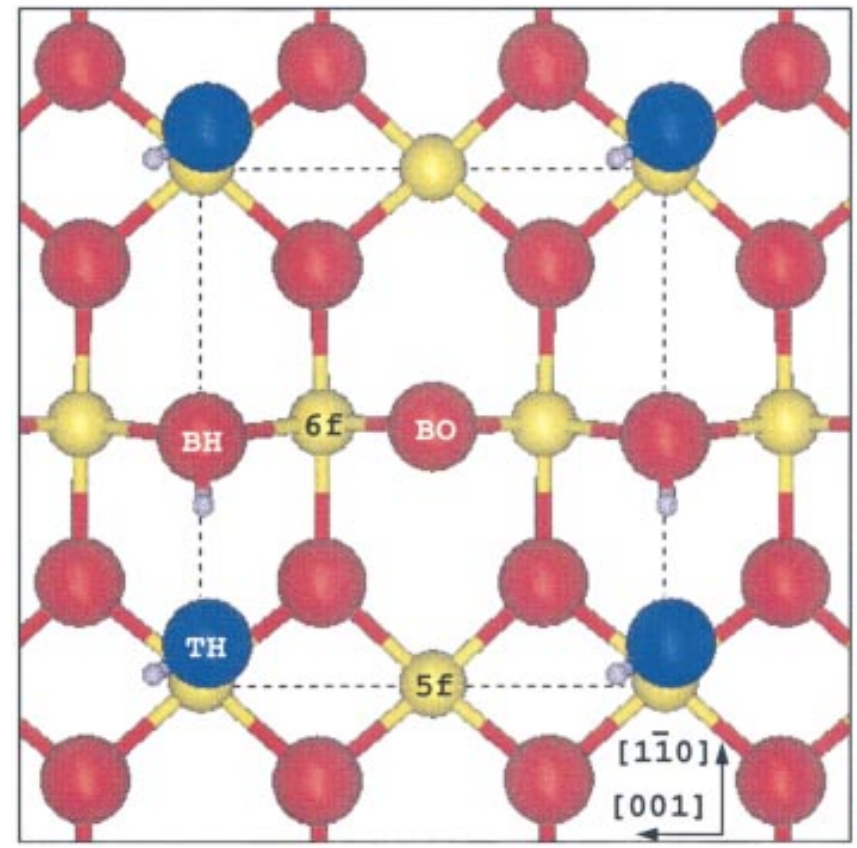

FIG. 1(color). Adsorption geometry at half-ML coverage. Red and yellow spheres denote surface $\mathrm{O}$ and $\mathrm{Ti}$, blue spheres adsorbate $\mathrm{O}$, and gray spheres $\mathrm{H}$. Dashed lines indicate the extent of the supercell. The labels are explained in the text.

vibrations. A variant of the CETEP code [29], the parallel version of CASTEP [12], was used for all calculations.

The initial configuration, shown in Fig. 1, is the fully relaxed structure obtained from the dissociative adsorption of a water molecule on one of the fivefold-coordinated $\mathrm{Ti}$ sites, giving a coverage of $0.5 \mathrm{ML}$. In the figure the symbols denote bridging-O, fivefold- and sixfoldcoordinated $\mathrm{Ti}(5 f$ and $6 f)$, "bridging hydroxyl," and "terminal hydroxyl." At this coverage the $5 f$ sites are the only ones at which water adsorbs strongly [11]. The first stage of our calculations was the exploration via MD of the adsorption of a second water molecule onto this surface. The natural second adsorption site is the $5 f \mathrm{Ti}$ not occupied by an $\mathrm{OH}$ group. Accordingly, we placed a second molecule above this site, oriented in the $1 \overline{1} 0$ plane with the $\mathrm{O}$ closest to the surface at $3 \AA$. However, we cannot rule out other adsorption sites a priori. We chose two other initial geometries for the second molecule, one above a $6 f \mathrm{Ti}$ atom and the other atop the bridging $\mathrm{OH}$ shown in the figure. In all three simulations the ions were given initial, random velocities corresponding to a temperature of $250 \mathrm{~K}$, and the adsorption process tracked for $0.75 \mathrm{ps.} \mathrm{Adsorption} \mathrm{occurred} \mathrm{only} \mathrm{above} \mathrm{the}$ $5 f$ site. In the other two cases, the second molecule drifted away from the surface. We take this as evidence that, at ML coverage, water is bound to $5 f \mathrm{Ti}$ sites. The nature of the adsorption is, however, surprising. The second molecule does not dissociate, but instead adopts a geometry in which hydrogen bonding to the terminal $\mathrm{OH}$ and the surface bridging oxygen takes place. This is stable throughout the duration of the MD run, and after subsequent relaxation to mechanical equilibrium. 
To check the stability of this state we have performed very accurate static calculations on the three most likely adsorption geometries. These are "mixed," the mixed mode just described; "2OH," both molecules dissociated; and " $2 \mathrm{M}$," both molecules intact. The mixed state was most stable, with an adsorption energy per molecule $E_{\text {mix }}=1.01 \mathrm{eV}$. The other states had adsorption energies of $E_{2 \mathrm{M}}=0.99$ and $E_{2 \mathrm{OH}}=0.91 \mathrm{eV}$. The relaxed geometry of the mixed state is shown in Fig. 2. The effect of the strong hydrogen bonding between the molecule, the terminal $\mathrm{OH}$, and the surface bridging oxygens is to reduce the adsorbate $\mathrm{O}-\mathrm{O}$ distance and to pull the adsorbates away from the $5 f$ sites. This effect is also apparent to a lesser degree in the $2 \mathrm{M}$ geometry.

Static calculations at half-ML coverage also help to explain the role of hydrogen bonding. The half-coverage dissociative adsorption energy $E_{\mathrm{OH}}$ of $0.91 \mathrm{eV} /$ molecule is identical to $E_{2 \mathrm{OH}}$. This leads to an important conclusion: At ML coverage there is effectively no interaction between $\mathrm{OH}$ groups on neighboring sites. This is supported by the fact that, in the $2 \mathrm{OH}$ system, there are no

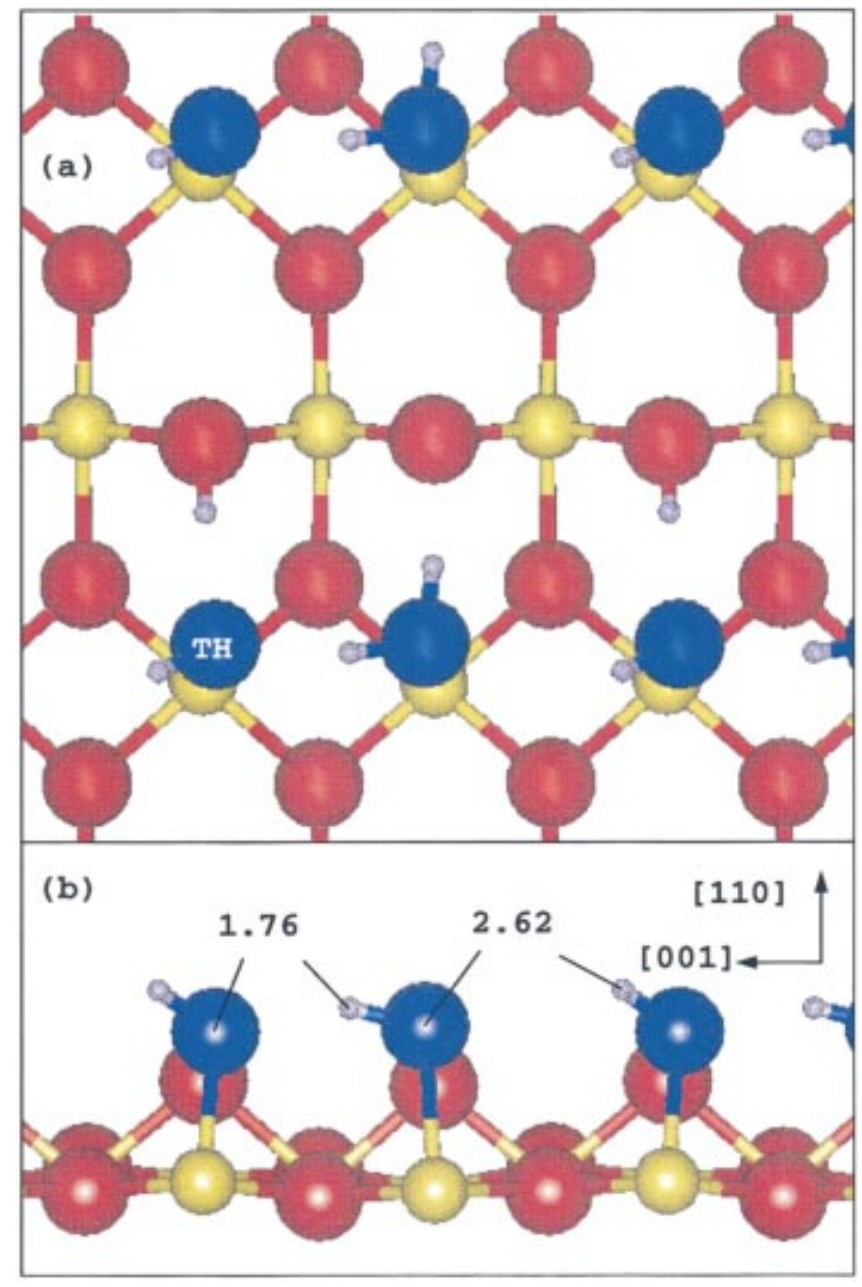

FIG. 2(color). Monolayer adsorbate configuration. Colors as in Fig. 1. (a) View from above the surface, and (b) side view along [1 $1 \overline{1} 0]$. The distances between $\mathrm{O}$ and $\mathrm{H}$ for the adsorbed $\mathrm{H}_{2} \mathrm{O}$ are indicated. symmetry-breaking relaxations as seen in the mixed and $2 \mathrm{M}$ states. The half-coverage molecular adsorption energy $E_{\mathrm{M}}$ is $0.87 \mathrm{eV} /$ molecule. Comparison with $E_{2 \mathrm{M}}$ reveals that $\mathrm{H}$ bonding contributes $0.12 \mathrm{eV} /$ molecule in the $2 \mathrm{M}$ state. Each molecule shares two $\mathrm{H}$ bonds with its neighbors. For the mixed state, subtracting $E_{\mathrm{OH}}$ and $E_{\mathrm{M}}$ from twice $E_{\text {mix }}$ shows that the H-bonding contribution is exactly the same, $0.12 \mathrm{eV} /$ molecule. However, in this case, the $\mathrm{H}$ of the terminal $\mathrm{OH}$ group is not involved in $\mathrm{H}$ bonding. This can be seen (i) by analogy with the $2 \mathrm{OH}$ system, where there are no intermolecular interactions, and (ii) from the vibrational analysis below. The additional binding arises solely from the short $\mathrm{H}_{2} \mathrm{O}$-terminal$\mathrm{OH}$ bond, which is, therefore, roughly twice as strong as the $\mathrm{H}$ bonds in the $2 \mathrm{M}$ geometry. Note also that at halfcoverage, only $0.04 \mathrm{eV}$ is gained through dissociation. This is why at ML coverage only half of the water dissociates: There is not enough energy to be gained by complete dissociation to offset the loss of all $\mathrm{H}$-bonding interactions. Of course, we have investigated only a $2 \times 1$ cell, and therefore cannot predict the fraction of molecules $f$ which will be dissociated. However, our results do suggest that at ML coverage some dissociation will occur, since the mixed state $(f=0.5)$ is lower in energy than the 2M state $(f=0)$.

The broad TPD peak observed between $250-320 \mathrm{~K}$ has been associated with the desorption of first-layer $\mathrm{H}_{2} \mathrm{O}$ [3]. This peak shifts downwards with increasing initial exposure. We expect that, under experimental conditions, the surface will support 1D chains of adsorbates at the $5 f$ sites separated by vacancies [30]. The calculated adsorption energies of 0.91 and $1.01 \mathrm{eV}$ (isolated $\mathrm{H}_{2} \mathrm{O}$ and ML coverage) correspond to the limits of shortand infinite-length chains. When analyzed via a simple Redhead analysis [11,31], these energies imply desorption in the range $270-300 \mathrm{~K}$. We suggest that the breadth of the peak and its shift with exposure arise from the variation in binding energy as a function of the length of these chains.

Finally, we present results from equilibrium MD calculations for the mixed state. After equilibration for $0.375 \mathrm{ps}$, we calculated the $\mathrm{H}$ velocity autocorrelation function over a $3 \mathrm{ps}$ run at $150 \mathrm{~K}$. The power spectrum of this function is shown in Fig. 3. Recall that the calculated frequencies are lowered because the $\mathrm{H}$ mass is $3 \mathrm{amu}$. Taking this into account, the features of the spectrum correspond closely to those observed in HREELS experiments [3], most notably the clear bond-bending signal at $29-30 \mathrm{THz}$ from the water molecule, and a broadened $\mathrm{OH}$ contribution at high frequencies. The sharp feature at $\sim 69 \mathrm{THz}$ is due to the vibrations of the $\mathrm{H}$ in the terminal $\mathrm{OH}$ alone: All other $\mathrm{H}$ ions contribute across the 50$70 \mathrm{THz}$ range. Since the broadening at high frequencies arises from $\mathrm{H}$ bonding [11], this underlines the fact that the $\mathrm{H}$ in the terminal $\mathrm{OH}$ group is not hydrogen bonded.

In summary, we propose that molecular and dissociated water can coexist on this surface, stabilized by a mutual hydrogen bond, but that single molecules with no 


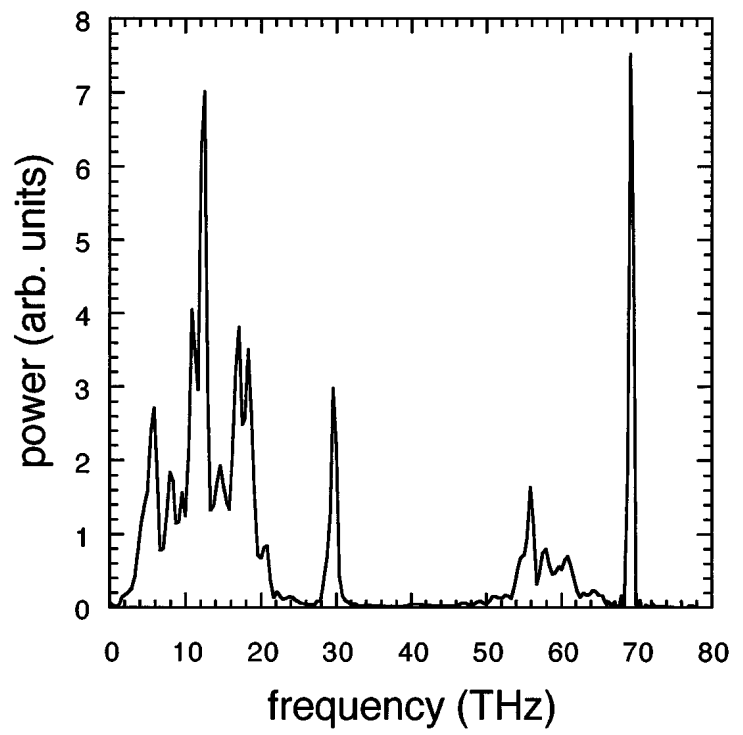

FIG. 3. Calculated hydrogen vibrational power spectrum for the mixed state at $150 \mathrm{~K}$.

immediate neighbors dissociate. As a corollary, molecular adsorption relies on $\mathrm{H}$ bonding. The strength of the hydrogen bonds depends on the adsorption mode (molecular or dissociative) of a molecule and its immediate neighbors. Competition with bonding to the surface results in a delicate balance between adsorption states. In addition, these intermolecular interactions influence the geometry of the adsorbates and their vibrational spectra. This scenario reconciles the majority of experimental data. It explains why dissociation occurs at low coverages, and is consistent with only a proportion of water being dissociated at ML coverage [4]. It also accounts for the broadening of the 270-K TPD peak. All of the features in HREELS spectra [3] are reproduced, and the small energy differences between mixed and totally molecular states indicate that the proportion of dissociated water may change with temperature, as suggested by Kurtz et al. [5]. Previous theoretical studies have failed to arrive at these conclusions because symmetry-constrained systems were studied. However, we have looked only at a $2 \times 1$ surface, and cannot predict the exact proportion of dissociated water at ML coverage. None of our conclusions are dependent on the presence of surface $\mathrm{O}$ defects. Finally, we note that the effects described will be very sensitive to changes in adsorbate spacing. Experiments or calculations of water adsorption on $\mathrm{SnO}_{2}$, which also has the rutile structure but whose lattice constants are some $6 \%$ larger, will be instructive since this will certainly change the balance between intermolecular and molecule-surface interactions.

This work is part of a CCP3/CCP5 joint project supported by Cray Research Inc. We are grateful for an allocation of time on the Cray T3D at EPCC provided by the High Performance Computing Initiative through the UKCP and Materials Chemistry consortia. Permission to use The DISPLAY graphics program (Paul
Sherwood, CCLRC Daresbury Laboratory) is gratefully acknowledged.

[1] V.E. Henrich and P. A. Cox, The Surface Science of Metal Oxides (Cambridge University Press, Cambridge, England, 1994).

[2] A. Fujishima and K. Honda, Nature (London) 238, 37 (1972).

[3] M. A. Henderson, Surf. Sci. 355, 151 (1996).

[4] M. B. Hugenschmidt, L. Gamble, and C. T. Campbell, Surf. Sci. 302, 329 (1994).

[5] R. L. Kurtz, R. Stockbauer, T.E. Madey, E. Román, and J. L. de Segovia, Surf. Sci. 218, 178 (1989).

[6] J. Goniakowski, S. Bouette-Russo, and C. Noguera, Surf. Sci. 284, 315 (1993).

[7] J. Goniakowski and C. Noguera, Surf. Sci. 330, 337 (1995).

[8] T. Bredow and K. Jug, Surf. Sci. 327, 398 (1995).

[9] A. Fahmi and C. Minot, Surf. Sci. 304, 343 (1994).

[10] J. Goniakowski and M.J. Gillan, Surf. Sci. 350, 145 (1996).

[11] P. J. D. Lindan, N. M. Harrison, J. M. Holender, and M. J. Gillan, Chem. Phys. Lett. 261, 246-252 (1996).

[12] M. C. Payne, M.P. Teter, D. C. Allan, T. A. Arias, and J. D. Joannopoulos, Rev. Mod. Phys. 64, 1045 (1992).

[13] G. P. Srivastava and D. Weaire, Adv. Phys. 36, 463 (1987).

[14] M. Parrinello, Solid State Commun. 102, 107 (1997).

[15] K. M. Glassford and J. R. Chelikowsky, Phys. Rev. B 47, 12550 (1993).

[16] M. Ramamoorthy, D. Vanderbilt, and R. D. King-Smith, Phys. Rev. B 49, 16721 (1994).

[17] J. Goniakowski, J. M. Holender, L. N. Kantorovich, M. J. Gillan, and J. A. White, Phys. Rev. B 53, 957 (1996).

[18] P. J. D. Lindan, N. M. Harrison, J. Holender, M. J. Gillan, and M. C. Payne, Surf. Sci. 364, 431 (1996).

[19] P. J. D. Lindan, N. M. Harrison, J. A. White, and M. J. Gillan, Phys. Rev. B 55, 15919 (1997).

[20] P. Jones and J. A. Hockey, Trans. Faraday Soc. 67, 2669 (1971); 67, 2679 (1971).

[21] C. A. Scamehorn, A.C. Hess, and M. I. McCarthy, J. Chem. Phys. 99, 2786 (1993).

[22] J. P. Perdew, in Electronic Structure of Solids '91, edited by P. Ziesche and H. Eschrig (Akademie Verlag, Berlin, 1991).

[23] J. P. Perdew, Phys. Rev. B 34, 7406(E) (1986).

[24] A. D. Becke, Phys. Rev. A 38, 3098 (1988).

[25] K. Laasonen, M. Sprik, M. Parrinello, and R. Car, J. Chem. Phys. 99, 9080 (1993).

[26] C. Y. Lee, D. Vanderbilt, K. Laasonen, R. Car, and M. Parrinello, Phys. Rev. B 47, 4863 (1993).

[27] J.P. Perdew, K. Burke, and M. Ernzerhof, ACS Symp. Ser. 629, 453 (1996).

[28] In fact, only slight differences in the energetics were found for the two systems, and relative stabilities were unchanged.

[29] L. J. Clarke, I. Štich, and M. C. Payne, Comput. Phys. Commun. 72, 14 (1992).

[30] We make the reasonable assumption that there is effectively no interaction between adsorbates along [1힝.

[31] P. A. Redhead, Vacuum 12, 203 (1962). 